\title{
MODELAGEM DO ERRO SISTEMÁTICO DE DISTÂNCIA NAS MEDIÇÕES REALIZADAS COM A CÂMARA PMD CAMCUBE 3.0
}

Systematic depth error modeling in range measurements on PMD CamCube 3.0

\author{
FELIPE MARTINS MARQUES DA SILVA \\ JORGE ANTONIO SILVA CENTENO
}

\author{
Programa de Pós-Graduação em Ciências Geodésicas \\ Universidade Federal do Paraná - Curitiba - Paraná - Brasil \\ felipemarques21@ hotmail.com \\ centeno@ufpr.br
}

\section{RESUMO}

As câmaras de distância são capazes de medir a distância entre o sensor e a superfície dos objetos para cada pixel da imagem. Comparando com os equipamentos de varredura a laser possuem a vantagem de obter a distância de vários pontos em um único instante, sem equipamento de varredura. As medidas obtidas pela câmara possuem erros sistemáticos que devem ser minimizados. Alguns fatores como o tempo de integração, a distância a ser medida, bem como a iluminação da cena, influenciam na obtenção da medida. Neste estudo foi analisada a influência da variação do tempo de integração e da distância câmara-alvo na exatidão do cálculo da distância, procurando modelar os erros sistemáticos das medições feitas com uma câmara PMD Camcube 3.0. A modelagem foi feita por meio da Transformada Discreta de Fourier e permitiu diminuir o erro médio quadrático (RMSE) de 15,01 cm para $5,05 \mathrm{~cm}$, para das observações feitas com tempo de integração de $4000 \mu$ s. Também foi verificado que a amplitude do erro é diretamente proporcional ao tempo de integração utilizado.

Palavras-chave: Erro de distância; Câmara de Distância; Tempo de Integração; Análise de Fourier. 


\section{ABSTRACT}

Range cameras are devices to measure the distance between the sensor and the object surface for each pixel within an image. Comparing to laser scanner they have the advantage of measuring range for several points at the same time without scanning systems. The measurements obtained by a range camera may have systematic errors that must be modeled to correct the measurements. Some factors as the integration time used, the distance to be measured, and the illumination of the scene, may affect the range measurement. In this paper we examine the influence of the integration time and the distance between the camera and the targets on the measured distance, proposing a model for the cyclic error of a Camcube PMD 3.0 camera using the Discrete Fourier Transform. The model allowed to reduce the root mean square error (RMSE) from $15.01 \mathrm{~cm}$ to $5.05 \mathrm{~cm}$ in observations made with the integration time of $4000 \mu \mathrm{s}$. It was also verified that the error amplitude is proportional to the integration time.

Key-words: Depth Error; Range Camera; Integration Time; Fourier Analysis.

\section{INTRODUÇÃO}

As câmaras de distância, também conhecidas por range cameras ou câmaras ToF (Time of Flight), são câmaras capazes de medir a distância entre o sensor e vários pontos (pixels da imagem) da superfície dos objetos de uma cena, em um único instante. Junto com a distância, a amplitude e a intensidade do sinal refletido pela superfície também são medidos, podendo a cena ser estática ou dinâmica (LICHTI, 2008).

Para calcular o valor das distâncias para cada pixel da imagem, a câmara de distância utiliza um dos dois métodos: (a) cálculo do tempo decorrido entre a emissão e o retorno de um pulso laser emitido pelo sensor (b) ou medição da diferença de fase entre a emissão e o retorno de um sinal de onda contínua emitido pelo sensor.

As medidas de distância, amplitude e intensidade dos pixels são obtidas em um único instante, sem a necessidade de mecanismos de varredura utilizados nos equipamentos de varredura a laser tradicionais (LICHTI, 2008; FOIX et. al., 2011; SHAHBAZI et. al., 2011). Segundo Lichti (2008) esta é a principal vantagem da câmara de distância sobre os equipamentos de varredura a laser. Outras vantagens sobre os equipamentos tradicionais de varredura a laser são: o baixo peso da câmara e o consumo reduzido de energia (FOIX et. al., 2011); a estrutura compacta com apenas um sensor para captar a informação de distância (FOIX et. al., 2011; SHAHBAZI et. al., 2011), diminuindo os problemas de oclusão; e a alta taxa de aquisição de imagens que possibilita o mapeamento e localização em tempo real (SHAHBAZI et. al., 2011). Lichti (2008) cita como desvantagens a baixa resolução e a limitação de distância livre de ambiguidades. 
É possível capturar vários instantes de um movimento - frequência de captura de quadros acima de 25 fps (frames per second, ou quadros por segundo) - como uma filmadora comum. Esta câmara pode ser utilizada em atividades como: reconhecimento de gestos ou movimentos humanos, para maior interação homemmáquina ou uso em jogos de vídeo; mobilidade de robôs, para reconhecer cenários e desviar de obstáculos; sensor para reconhecimento de pedestre em via pública, para evitar atropelamentos ou colisão com automóveis; "reconstrução" do movimento executado por um atleta profissional, para melhorar a execução do movimento, etc.

As medidas obtidas com as câmeras são eivadas de erros, originados tanto por fatores ambientais, bem como não ambientais. Alguns fatores interferem no cálculo da distância são: a iluminação da cena, o tempo de integração, a frequência e a amplitude do sinal emitido, as características refletivas dos objetos, a temperatura interna da câmara, a própria distância câmara/objeto.

No presente artigo foi utilizada a câmara PMD Camcube 3.0, capaz de obter imagens de intensidade, distância e amplitude, com 200 × 200 pixels (40.000 pixels). Este equipamento utiliza o princípio time-of-flight (ToF) com base no método de diferença de fase, para medir a distância para cada pixel de uma imagem. Foi verificada a influência da variação do Tempo de Integração e da distância câmara-alvo na exatidão do cálculo da distância, procurando modelar o erro cíclico observado nas medições por meio da Transformada Discreta de Fourier.

Convém esclarecer que no desenvolver deste artigo são citados os termos amplitude e frequência. Entretanto, deve-se distinguir entre a amplitude e frequência de modulação do sinal emitido pela câmera, e a amplitude e frequência do erro cíclico observado pela variação da distância câmera/alvo e do tempo de integração.

\section{ESTADO DA ARTE}

O cálculo de distâncias nas câmeras TOF é influenciado por erros originados por fontes que dependem das características do ambiente, bem como por erros originados por fontes que não dependem das características do ambiente.

Quanto às fontes de erro que não dependem das características do ambiente também chamados erros instrumentais - pode-se citar: a temperatura do sensor (KAHLMANN et. al., 2008; STEIGER et. al., 2008; CHIABRANDO et. al. (2009); PIATTI e RINAUDO, 2012); o tempo de integração (KAHLMANN et. al., 2006; WIEDEMANN et. al., 2008; FOIX et. al., 2011) e; os materiais de fabricação do sensor (LANGE, 2000; KAHLMANN et. al., 2006; LINDNER E KOLB, 2007; FOIX et. al., 2011).

Já as fontes de erro que dependem das características do ambiente, tem-se: a relação sinal-ruído (FOIX et. al., 2011; LIU, 2002); a amplitude da reflexão dos objetos da cena (LINDNER e KOLB, 2007; FALIE e BUZULOIU, 2007; FOIX et. al., 2011); o multi-caminho que o sinal percorre entre a emissão e o retorno (FUCHS, 2012); o espalhamento interno do sinal na câmera (LINDNER E KOLB, 2007; KAREL, 2008; SABOV e KRÜGER, 2008; MAY et. al., 2009; REYNOLDS 
et. al., 2011) e; a iluminação ambiente (MÖLLER et. al., 2005; KAHLMANN, 2007; MAY et. al., 2007).

Além das fontes de erros já citadas - as que dependem e as que não dependem das características do ambiente - sabe-se de outras duas fontes de erro: o ângulo de incidência do sinal sobre o alvo (HEBERT e KROTKOV, 1992; ANDERSON et. al., 2005; KIM et. al., 2008) e; a distância entre a câmera e o alvo (LINDNER et. al., 2010; KOLB et. al. 2010; FOIX et. al., 2011).

O presente artigo trata do erro ocasionado pela distância entre a câmera e o alvo. É um erro sistemático que pode ser visualizado mediante um gráfico que relacione a distância medida pela câmera e a distância admitida como verdadeira, onde se observa que tende a uma curva senoidal (ver Figura 1).

Figura 1 - Erro de distância: erro da medida vs. distância real.

Fonte: Foix et. al. (2011).

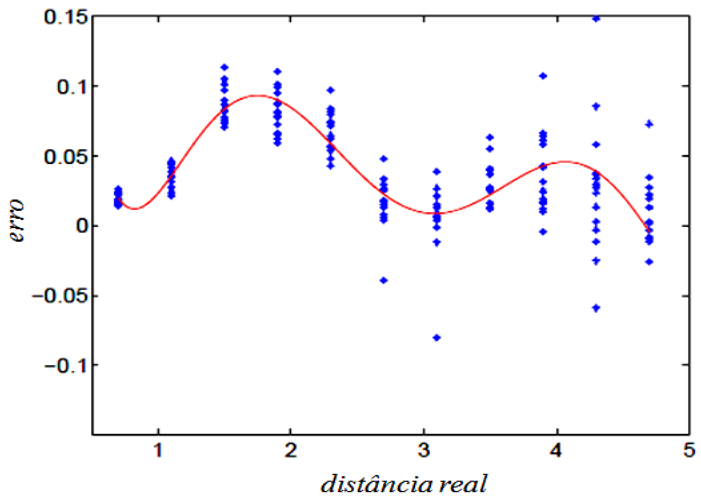

Segundo Lindner et. al. (2010), Kolb et. al. (2010) e Foix et. al. (2011), este erro ocorre devido à limitações tecnológicas e de custo, de modo que há uma incapacidade de modular perfeitamente sinal emitido segundo um modelo matemático teórico pré-estabelecido utilizado pela câmera - geralmente senoidal.

Diversos pesquisadores procuraram minimizar este erro por meio de abordagens como: regressão linear, look-up table, ajustamento de observações com uso de B-splines, funções polinomiais, e algoritmos de modulação/demodulação. Algumas destas pesquisas são citadas nos parágrafos abaixo.

Kuhnert e Stommel (2006), durante a calibração de uma câmera, utilizaram um modelo linear para minimizar o erro de distância, considerando uma janela central 5x5. Também modelaram o desvio padrão por meio de uma função de $2^{\circ}$ grau, uma vez que a iluminação decai em função do quadrado da distância.

Já Lindner e Kolb (2006) utilizaram o ajustamento por mínimos quadrados com uma curva B-spline para realizar a calibração de distância, desde $3.75 \mathrm{~m}$ até $7.5 \mathrm{~m}$. Dentre outros trabalhos onde foram utilizadas curvas B-splines pode-se citar 
Kahlman et.al. (2006), Fuchs e Hirzinger (2008), Lindner (2010) e Lindner et. al. (2010).

Também ao realizar a calibração da distância, Khalmann et. al. (2007) utilizaram da técnica look-up table (LUT) juntamente com uma matriz FPN (fixed pattern noise), que tem por objetivo corrigir erros devidos aos materiais utilizados na fabricação do sensor. Dentre outros autores que fizeram uso da técnica lookup table pode-se citar Radmer et. al. (2008) e Hussman et. al. (2012).

Trabalhos de Kim et. al. (2008) e Schiller et. al. (2008) realizaram a correção de distância utilizando funções polinomiais de $3^{\circ}$ grau e $6^{\circ}$ grau, respectivamente.

Lindner et. al (2008) corrigiram os erros de distância através de um novo algoritmo de demodulação e da consequente amostragem da função de correlação. Consideraram que o sinal emitido é retangular, baseado-se no fato de que, por limitações tecnológicas e de custo, não é viável emitir um sinal perfeitamente senoidal. Já Hussmann e Knoll (2013) desenvolveram um equipamento de iluminação que modula uma onda retangular emitida pela câmera de distância em uma onda senoidal. Posteriormente Hussmann et. al. (2014) desenvolveram um modelo que considera a distribuição de ruídos na imagens de distância, visando atenuar o erro de distância.

Diante do exposto, no presente artigo a correção dos erros de distância foi realizada utilizando a Transformada Rápida Discreta de Fourier e a equação senoidal / cossenoidal da onda.

\section{FUNDAMENTAÇÃO TEÓRICA}

\subsection{Câmaras TOF - Princípios}

A câmara PMD Camcube 3.0 utiliza o princípio Time-of-Flight (ToF) e mede a distância usando o princípio de onda contínua, onde a distância é calculada com base na diferença de fase entre um sinal modulado de onda emitido e o sinal demodulado recebido. A modulação consiste em um processo de alteração da frequência e/ou da amplitude de uma onda eletromagnética, para transmitir um sinal em uma onda portadora. Já a demodulação (ou detecção) consiste no processo inverso, isto é, detectar a onda portadora e extrair o sinal modulado. Uma onda portadora é caracterizada por três variáveis: amplitude, frequência e fase.

Na Figura 2 são mostradas duas ondas senoidais representando o sinal emitido $\mathrm{g}(\mathrm{t})$, em linha cheia, e o sinal recebido ou de retorno $\mathrm{s}(\mathrm{t})$, em linha pontilhada. A amplitude do sinal de retorno (A) tende a ser menor que a do sinal emitido, uma vez que apenas parte do sinal retorna ao sensor de câmara. $O$ valor da amplitude do retorno influencia a qualidade do valor da distância calculada. Na mesma Figura 2 é mostrada a diferença de fase $(\varphi)$ entre os sinais, e o comprimento de onda $(\lambda)$. 
Figura 2 - Esquema para representação da diferença de fase $(\varphi)$.

Fonte: Adaptado de Piatti (2010).

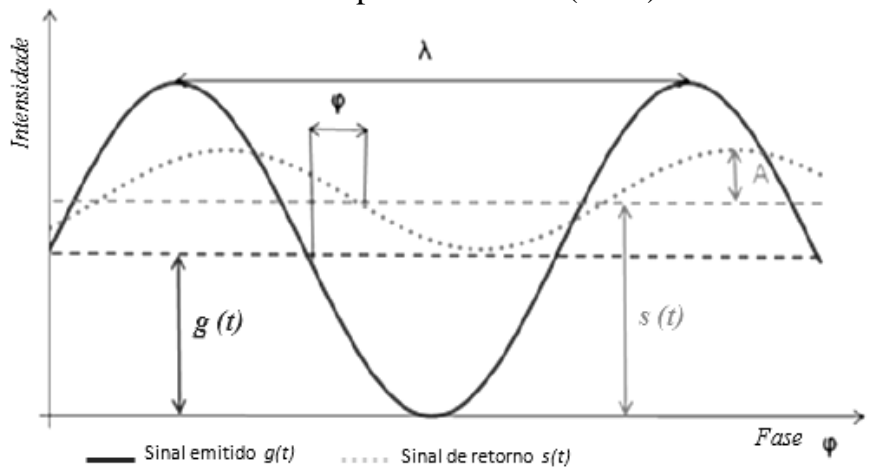

Sendo $g$ (t) o sinal emitido - modulado, ao mesmo tempo, em amplitude e frequência - pelas fontes de iluminação da câmara, represenFtado matematicamente pela Equação 1, e s(t) o sinal recebido que incide sobre o sensor, representado pela Equação 2, as distâncias para cada pixel são calculadas com base em uma função de correlação cruzada (FC) entre estes dois sinais. Esta correlação cruzada, representada na Equação 3, é utilizada para medir o atraso entre o sinal emitido e o sinal recebido, que corresponde à diferença de fase entre os dois sinais (LICHTI, 2008; LINDNER e KOLB, 2006).

$$
\begin{gathered}
g(t)=\cos (\omega-t) \\
s(t)=k+A \cdot \cos (\omega-t+\varphi) \\
c(\tau)=(s \star g)(\tau)=I+\frac{A}{2} \cdot \cos (\omega-t+\varphi)
\end{gathered}
$$

sendo: $\mathrm{g}(\mathrm{t})=$ função do sinal modulado emitido;

$\mathrm{s}(\mathrm{t})$ = função do sinal recebido (ou sinal de retorno);

$c(\tau)=$ função de correlação entre os sinais emitido e recebido;

$\mathrm{A}=$ amplitude do sinal recebido, utilizado na imagem de amplitude;

$\mathrm{I}=$ intensidade do sinal recebido (offset);

$\tau=$ diferença interna de fase;

$\star=$ operador de correlação cruzada;

$\mathrm{k}$ = componente não modulada, decorrente da iluminação ambiente;

$\omega=$ frequência angular de modulação do sinal emitido $\mathrm{g}(\mathrm{t})$;

$\varphi=$ diferença de fase entre o sinal emitido $\mathrm{g}(\mathrm{t})$ e o sinal recebido $\mathrm{s}(\mathrm{t}) \quad$; $\mathrm{t}=$ tempo. 
Para o cálculo da diferença de fase, são tomadas quatro amostras da função de correlação, em instantes distintos $-\mathrm{c}\left(\tau_{0}\right), \mathrm{c}\left(\tau_{1}\right), \mathrm{c}\left(\tau_{2}\right)$ e $\mathrm{c}\left(\tau_{3}\right)-$ defasados de $\pi / 2$ ao longo do tempo, conforme pode ser visto na Figura 3. A diferença de fase é então calculada pela Equação 4 (KHALMANN et. al., 2006).

Figura 3 - Esquema para representação da diferença de fase $(\varphi)$.

Fonte: Adaptado de Becerro (2008).

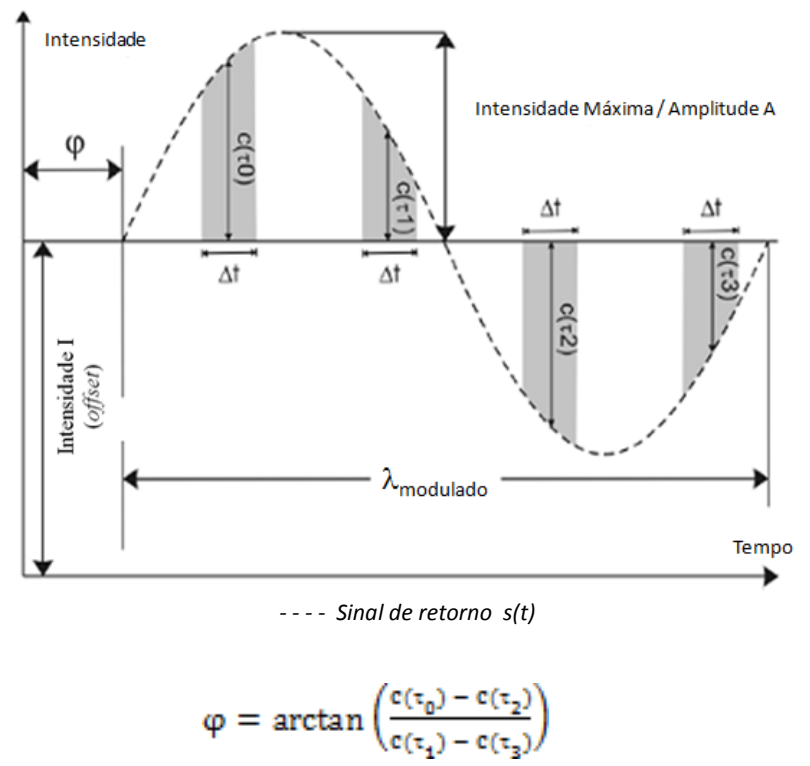

Com o valor da diferença de fase pode-se calcular a distância (D) com a Equação 5,

$$
\mathrm{D}=\frac{\mathrm{c} \cdot \varphi}{4-\pi \cdot \omega}+\mathrm{N}-\lambda
$$

onde: $\mathrm{c}=$ velocidade da luz;

$\mathrm{N}$ = número de ciclos inteiros;

$\lambda=$ comprimento de onda;

$\omega=$ frequência angular do sinal emitido $\mathrm{g}(\mathrm{t})$;

$\varphi=$ diferença de fase entre o sinal emitido $g(t)$ e o sinal recebido $s(t)$.

$\mathrm{O}$ termo $\mathrm{N}$ representa o número de ciclos inteiros, e é chamado de ambiguidade. A primeira parte da equação é a parte fracionada do sinal e é a única utilizada para calcular as distâncias. Pois, não sendo possível determinar o número de ciclos inteiros, o termo $\mathrm{N}$ é desconsiderado. Esta opção por desconsiderar a 
ambiguidade N, limita a distância de utilização de câmera ao valor de meio comprimento de onda. Considerando que o sinal é emitido à velocidade da luz e com frequência de $20 \mathrm{MHz}$, o comprimento de onda será de $15 \mathrm{~m}$. Como o sinal tem que percorrer a distância câmara/alvo na ida e na volta, a distância máxima, livre de ambiguidades, entre o sensor e o alvo fica limitada a 7,5m.

O cálculo da distância para cada pixel, por fim, é feito utilizando a Equação 6.

$$
\mathrm{D}=\frac{\mathrm{c}^{*} \varphi}{4 \cdot \pi^{*} \omega}
$$

Outras grandezas que podem ser calculadas são: a intensidade, para as imagens de intensidade; e a amplitude, para as imagens de amplitude.

Os valores da intensidade (I) e da amplitude (A) são calculados pela Equação 7 e pela Equação 8, respectivamente (KHALMANN et. al., 2006).

$$
\begin{gathered}
I=\frac{c\left(\tau_{0}\right)+c\left(\tau_{1}\right)+c\left(\tau_{2}\right)+c\left(\tau_{2}\right)}{4} \\
A=\frac{\sqrt{\left[c\left(\tau_{0}\right)-c\left(\tau_{2}\right)\right]^{2}+\left[c\left(\tau_{1}\right)-c\left(\tau_{1}\right)\right]^{2}}}{2}
\end{gathered}
$$

sendo:

$\mathrm{I}=$ intensidade do sinal de retorno $\mathrm{s}(\mathrm{t})$ (offset);

$\mathrm{A}=$ amplitude do sinal de retorno $\mathrm{s}(\mathrm{t})$;

$\mathrm{c}\left(\tau_{0}\right), \mathrm{c}\left(\tau_{1}\right), \mathrm{c}\left(\tau_{2}\right), \mathrm{c}\left(\tau_{\mathrm{g}}\right)=$ amostras do sinal de retorno, defasadas de $\pi / 2$.

\subsection{Erro Sistemático}

Segundo Landgate (2009), nos equipamentos de princípio EDM distinguem-se três tipos de erros sistemáticos instrumentais: erro de zero, erro de escala, e erro cíclico. Erros sistemáticos ocorrem em estações de medição com princípio EDM, bem como em câmaras de distância (LICHTI, 2008; LINDNER e KOLB, 2006).

\subsubsection{Erro de Zero}

É a diferença entre o centro geométrico do instrumento - materializado pela interseção dos eixos vertical, horizontal, e eixo de colimação - e o centro eletrônico do instrumento (FAGGION, 2001). O centro eletrônico é o ponto onde parte o sinal que medirá a distância. Este erro também é chamado de constante aditiva, pois seu valor deve ser somado às distâncias medidas. 
Para determinar o valor do erro de zero $\left(\mathrm{Z}_{0}\right)$, deve-se medir três distâncias $\mathrm{L}_{1}, \mathrm{~L}_{2}$ e $\mathrm{L}_{3}$ - com o instrumento eletrônico desejado, obtendo as distâncias medidas $\mathrm{d}_{1}, \mathrm{~d}_{2}$ e $\mathrm{d}_{3}$, conforme a Figura 4 .

Figura 4 - Distâncias para determinação do valor do Erro de Zero. Fonte: Adaptado de Gripp (1986).
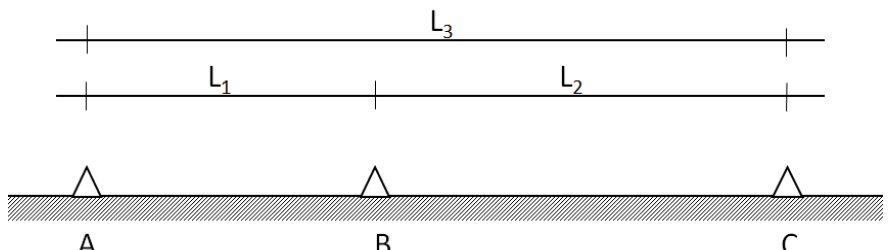

A

C

Com base nestas três medidas e utilizando as Equações 10 e 11, determina-se o valor de $\mathrm{Z}_{0}$ (FAGGION, 2001).

$$
\begin{array}{r}
\left(d_{3}+Z_{0}\right)=\left(d_{1}+Z_{0}\right)+\left(d_{2}+Z_{0}\right) \\
Z_{0}=d_{3}-d_{1}-d_{2}
\end{array}
$$

\subsubsection{Erro de Escala}

Este erro ocorre quando a frequência modulada no momento da emissão do sinal não corresponde exatamente à frequência projetada para o aparelho (MAIA, 1999). Esta alteração no valor da frequência é corrigida por meio da Equação 12, sendo que as distâncias são corrigidas pela Equação 13 (FAGGION, 2001).

$$
\begin{gathered}
c=\frac{\left(f_{n}-f_{m}\right)}{f_{m}} \\
d_{c}=d_{m}+\frac{\left(f_{n}-f_{m}\right)}{f_{m}} \cdot d_{m}
\end{gathered}
$$

onde:

$\mathrm{C}=$ correção $\left(\right.$ em ppm); $\quad \mathrm{d}_{\mathrm{c}}=$ distância corrigida; $\quad \mathrm{d}_{\mathrm{m}}=$ distância medida; $\quad \mathrm{f}_{\mathrm{n}}=$ frequência nominal do instrumento; $\quad \mathrm{f}_{\mathrm{m}}=$ frequência medida.

\subsubsection{Erro Cíclico}

O Erro Cíclico ocorre no processo de medição interna da diferença de fase, devido à diferença de superposição entre o sinal emitido e o sinal recebido (SURVEYOR - GENERAL VICTORIA, 2012). 


\subsection{Tempo de Integração ( TI )}

O tempo de integração (TI) consiste no intervalo de tempo disponível para o sensor captar a luz incidente no sensor, sendo similar à velocidade do obturador das câmeras analógicas. A mudança do tempo de integração da câmara afeta os valores de distância em todos os pixels da imagem (FOIX et. al., 2011). O valor do pixel é proporcional ao tempo de integração, podendo ter valores nulos devido à baixa exposição à luz, até valores saturados devido ao excesso de exposição à luz. Ambas as situações acarretam erros nas medidas (OLIVEIRA, 2011).

\section{EQUIPAMENTOS}

A seguir são mostrados os equipamentos utilizados neste estudo: a câmara de distância, o interferômetro laser, e o alvo móvel.

\subsection{Câmara PMD CamCube 3.0}

Foi utilizada uma Câmara PMD CamCube 3.0, mostrada na Figura 5, com fontes de iluminação de LED acopladas nas laterais. Cada uma das fontes de iluminação possui 48 diodos emissores de luz chamados de LEDs (Light Emitting Diodes), que emitem luz infravermelha com comprimento de onda de $870 \mathrm{~nm}$. Utiliza um sensor de imageamento PMD (Photonic Mixer Device), da empresa PMD Technologies, que consiste em um chip, 200x200 pixels, de tecnologia CMOS padrão, mas com a capacidade de armazenar a distância de cada pixel.

Figura 5 - PMD CamCube 3.0 com iluminação LED nas laterais, sobre tripé. Fonte: Lichti et. al. (2013).

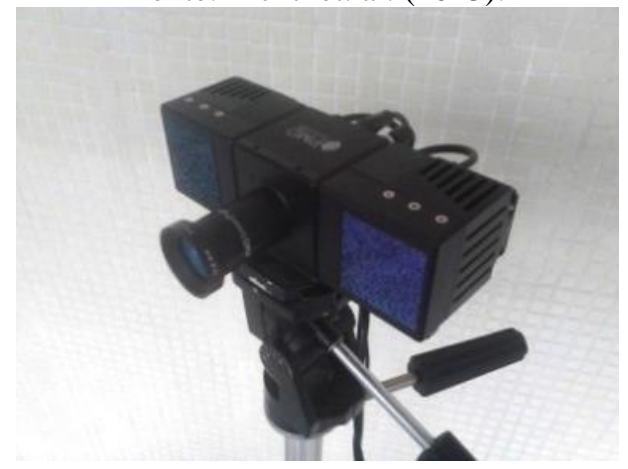

A distância entre o sensor e o alvo é obtida pelo método da diferença de fase, correlacionando o sinal emitido (modulado em amplitude e frequência) e o sinal de retorno ou refletido (demodulado), assumindo que este sinal de retorno possui a forma senoidal. (OLIVEIRA et. al., 2010). O Quadro 1 apresenta as principais especificações técnicas da PMD Camcube 3.0 
Quadro 1 - Principais especificações técnicas da PMD Camcube 3.0.

Fonte: Piatti (2010).

\begin{tabular}{|l|l|}
\hline Sensor & CMOS 200 x 200 pixels \\
\hline Distância focal & $12.8 \mathrm{~mm}$ \\
\hline Campo de Visão & $40^{\circ}(\mathrm{h}) \times 40^{\circ}(\mathrm{v})$ \\
\hline Comprimento de onda da iluminação padrão & $870 \mathrm{~nm}$ \\
\hline Range & de $0,3 \mathrm{~m}$ a 7,5m \\
\hline Taxa de aquisição de imagens (frame rate) & $40 \mathrm{fps}$ \\
\hline Acurácia absoluta & não disponível \\
\hline Tamanho do pixel & não disponível \\
\hline
\end{tabular}

Além das característica citadas no Quadro 1, a câmara PMD Camcube 3.0 permite modular o sinal com nas frequências $18 \mathrm{MHz}, 19 \mathrm{MHz}, 20 \mathrm{MHz}$ e $21 \mathrm{MHz}$. A amplitude do sinal é modulada em um padrão da câmera, não podendo ser alterada. Mas é possível alterar o tempo de integração da câmara, de 12 a $50000 \mu$ s.

\subsection{Interferômetro Laser}

Para obter um conjunto de medidas de referência com maior exatidão e precisão, foi utilizado um interferômetro laser (Hewlett-Packard, Modelo 5508A). Este interferômetro possui trilhos metálicos com, aproximadamente, 4,2 metros de comprimento, sendo capaz de obter medidas com precisão de até $10^{-8} \mathrm{~m}$. Sendo que esta precisão é superior à necessária para as correções das distâncias medidas pela câmara de distância, optou-se por medir com precisão de $10^{-5} \mathrm{~m}$.

\subsection{Alvo}

O alvo utilizado constituiu-se de uma chapa de madeira compensada, de dimensões $25 \times 60 \mathrm{~cm}$, pintada na cor branca. Está apoiado sobre o refletor móvel do interferômetro, sendo possível alterar as distâncias entre a câmara e o alvo.

Experimentos de Lindner e Kolb (2007) e Lindner et. al. (2010) utilizaram um tabuleiro composto de quadrados pretos e brancos, onde foi verificado que a diferença de energia refletida entre os quadrados brancos e os quadrados pretos interfere no cálculo da distância. Isto porque a amplitude do sinal refletido pelos quadrados brancos será maior, e a variação da amplitude causa variação no cálculo da distância. Visto que tal ocorrência não era desejada, optou-se por um alvo de cor única, branca, para possibilitar a mesma reflectância em toda a superfície do alvo.

\section{METODOLOGIA}

\subsection{Aquecimento do sensor da câmera}

Estudosde Kahlmann et. al. (2006), Steiger et. al. (2008), Chiabrando et. al. (2009), e Piatti e Rinaudo (2012), entre outros, indicam a necessidade de estabilizar as medições feitas pela câmara, mediante o aquecimento da câmara e do seu sensor. 
Caso sejam tomadas imagens antes deste tempo, as medições terão muitas variações, sendo pouco confiáveis.

Segundo estudos de Kahlmann et. al. (2006) com a câmara SwissRanger ${ }^{\text {TM }}$ SR2, depois de passados 04 (quatro) minutos com a câmara ligada, as medições realizadas com a câmara estabilizam, isto é, apresentaram valores com baixa variância. Steiger et. al. (2008), utilizando a câmara SwissRanger SR-3000, também obteve valores estáveis após pouco mais de 4 minutos (250 segundos) de espera. Já Chiabrando et. al. (2009), utilizando uma câmara SwissRanger SR-4000, realizou experimentos onde foram necessários 40 (quarenta) minutos para estabilizar os valores das medições. Piatti e Rinaudo (2012), utilizando a câmara PMD Camcube 3.0, informam que é suficiente aguardar 40 minutos com a câmara ligada para atingir estabilidade nas medições. Sendo assim, a câmara é ligada durante o tempo necessário para estabilizar os valores de distância, daí então se obtém as imagens.

\subsection{Tomada de Imagens}

Inicialmente espera-se o tempo necessário para estabilizar as medidas obtidas com a câmera. No item 6, "Experimentos e Análise de Resultados", é mostrado um experimento realizado com a câmara PMD para verificar o tempo de aquecimento do sensor.

Com a câmara instalada sobre um tripé foram coletadas imagens de distânciado alvo, em distâncias pré-estabelecidas, medidas por meio do interferômetro. A frequência de modulação do sinal infravermelho emitido foi fixada em $20 \mathrm{MHz}\left(20 \cdot 10^{6} \mathrm{~Hz}\right)$. Foram tomadas imagens em 41 posições sobre um trilho metálico, distantes $10 \mathrm{~cm}$ entre si, desde a posição inicial $70 \mathrm{~cm}$ até a posição final $470 \mathrm{~cm}$. Em cada posição foram tomadas imagens de distância para tempos de integração (TI) desde $\mathrm{TI}=100 \mu$ s até $\mathrm{TI}=4000 \mu \mathrm{s}$, variando a cada $100 \mu \mathrm{s}$.

Esta grande quantidade de imagens (medidas de distância) possibilita verificar a variação dos erros mais detalhadamente. Também variou-se o tempo de integração para verificar sua influência nas medidas obtidas com a câmara. A Figura 6 mostra um desenho esquemático do experimento, onde se pode observar: as posições da câmara PMD Camcube 3.0 e do interferômetro laser; o display de medições do interferômetro; o alvo apoiado sobre o refletor móvel; os trilhos metálicos; e uma escala com as 41 posições para tomada de imagens. 
Figura 6 - Desenho esquemático da coleta de imagens com a câmara PMD CamCube 3.0, utilizando interferômetro laser.

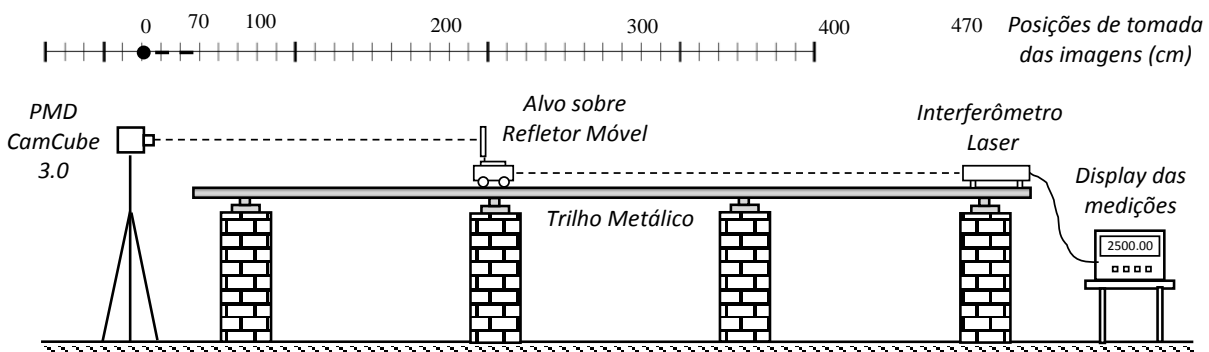

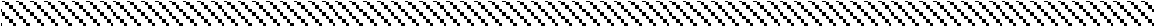

Inicialmente seria analisado apenas o pixel central das imagens. Entretanto, para não depender apenas de um valor de pixel a cada distância - pois este valor pode apresentar maiores erros em relação ao valor real - optou-se por analisar os valores dos pixels de uma janela 7 × 7 no centro do alvo e a partir deles calcular uma média para cada posição do alvo.

As Figuras $7 \mathrm{a}$ e $7 \mathrm{~b}$ mostram a câmara e o alvo sobre o refletor móvel do interferômetro, que é deslocado ao longo dos trilhos. O processo de medição consiste em posicionar o alvo ao longo dos trilhos, medir a distância de referência usando o interferômetro e obter uma série de imagens para cada posição, variando o tempo de integração.

De posse das imagens, fez-se um programa em ambiente Matlab para leitura de uma região de $7 \times 7$ pixels no centro da imagem contendo o alvo. Estes dados foram usados para calcular a média aritmética e o desvio padrão desta região. A média e o desvio padrão tomados nestes 49 pixels servem para se obter um valor de distância mais confiável. Desvio padrão alto indica que algum pixel na janela foi capturado com valor de distância discrepante.

Figura 7 - Fotos do experimento:(1) câmara sobre tripé; (2) alvo sobre o refletor móvel do interferômetro; (3) alvo na posição mais distante.
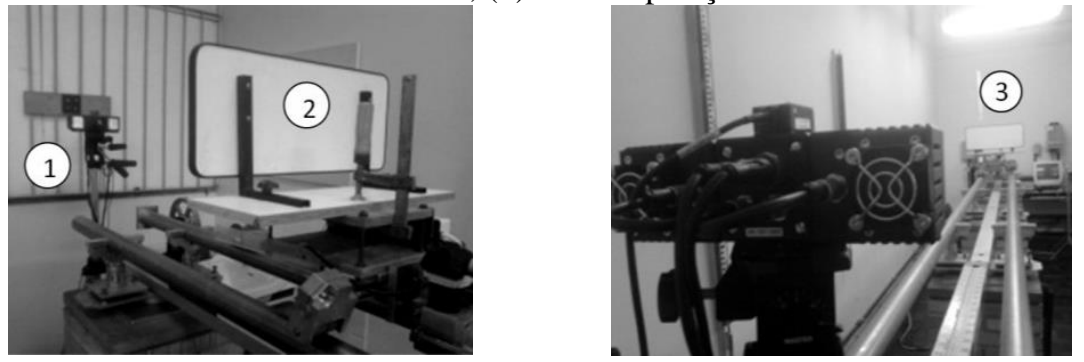

Bol. Ciênc. Geod., sec. Artigos, Curitiba, v. 21, nำ1, p.126-148, jan-mar, 2015. 


\subsection{Aplicação da Transformada de Fourier e da Função de Correção}

Tendo as distâncias medidas em cada posição, utilizou-se a Transformada Discreta de Fourier para determinar as principais frequências sobre os valores dos erros e assim aproximar uma função ao erro cíclico.

A Transformada de Fourier permite analisar uma onda (sinal) qualquer no espaço temporal, decompondo-a em suas componentes harmônicas - seno e cosseno - com suas diferentes frequências e amplitudes no espaço espectral. Aplicando este conceito às funções contínuas, é possível representar uma função de forma aproximada com a combinação de funções harmônicas. Quanto mais harmônicos de diferentes frequências e amplitudes forem considerados, melhor será a aproximação.

A Transformada de Fourier pode ser representada num espaço discreto, sendo conhecida como Transformada Discreta de Fourier. Utilizando um número finito de pontos no domínio do tempo, tem-se uma representação discreta do sinal no domínio da frequência. A função da transformada discreta de Fourier e sua inversa são representadas, respectivamente, pelas Equações 14 e 15:

$$
\begin{aligned}
& F(f)=\frac{1}{N} \sum_{t=0}^{N-1} s(t)-e^{\left(\frac{-12 \pi \pi f 5}{N}\right)} \\
& F^{-1}(F(f))=\sum_{\mathscr{W}=0}^{N-1} F(\omega)-e^{\left(\frac{-1 \mathrm{z} \pi \mathrm{m}}{N}\right)}
\end{aligned}
$$

$\omega=$ frequência angular do sinal, onde $\omega=2 \pi \cdot f ; \quad f=$ frequência do sinal ;

$\mathrm{e}=$ número de Euler (número neperiano).

A função de correção dos erros é a equação da onda (Equação 16), de movimento harmônico amortecido, pois, como o erro diminui com a distância - isto é, a amplitude é atenuada conforme a distância aumenta - a atenuação desta amplitude foi modelada usando uma função linear (Equação 17). Para isto, considerou-se apenas o valor absoluto dos mínimos e máximos do erro observado e uma reta foi aproximada. Outras funções poderiam ser usadas, porém a função linear foi preferida pelo fato de ter um número reduzido de picos. Finalmente, duas constantes foram estimadas para modelar o deslocamento relativo entre as funções, tanto em amplitude, como em distância.

$$
\begin{gathered}
y(x)=c+A \cdot \cos (2 \cdot \pi \cdot f \cdot x+\varphi) \\
A(x)=m \cdot x+n
\end{gathered}
$$

onde:

$\mathrm{m}=$ declividade da reta; $\mathrm{n}=$ constante da reta; 
$\mathrm{x}=$ posição ou distância; $\quad \mathrm{c}=$ constante da onda;

$\mathrm{A}=$ amplitude; $\quad f=$ frequência do sinal; $\quad \varphi=$ diferença de fase.

\section{EXPERIMENTOS E ANÁLISE DOS RESULTADOS}

Inicialmente foi realizado um experimento para avaliar o tempo necessário de pré-aquecimento do sensor da câmara. Verificando a variação dos valores da distância em função do tempo de inicialização, é possível determinar o tempo necessário para atingir a estabilização da temperatura do sensor. Para isso, a câmara foi posicionada na frente do alvo e foram monitorados os valores de distância e o desvio padrão da região central da imagem ao longo de uma hora.

Nota-se na Figura 7, que os valores iniciais da distância são pequenos e eles crescem na medida em que o tempo passa, ou seja, o sensor se aquece. Após 40 minutos, o sensor atinge um estado estável e as medidas de distância não sofrem variações significativas. O mesmo não ocorre com o desvio padrão, o qual se mantém estável ao longo do período de análise, como pode ser visto na Figura 8. Isto indica que a temperatura do sensor não afeta a precisão das medidas, mas sim sua exatidão, gerando estimativas menores que o valor real quando o sensor não está aquecido. Tais resultados concordam com os de Piatti e Rinaudo (2012).

Neste estudo foi efetuado o pré-aquecimento do sensor por aproximadamente 50 minutos. Estabilizada a temperatura do sensor da câmara, iniciou-se a tomada das imagens e a obtenção da distância média da janela central 7x7 pixels, através de programas desenvolvidos em Matlab.

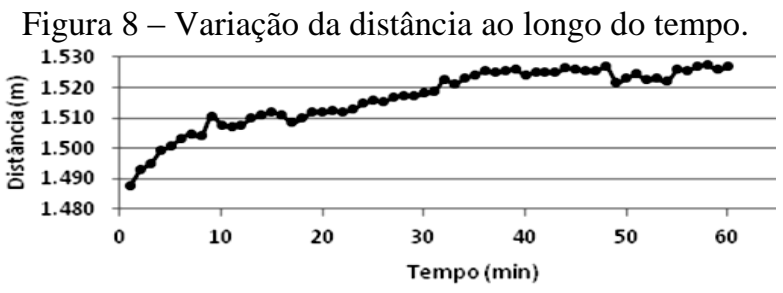

A Figura 8 mostra a variação da distância do pixel central ao longo do tempo e a Figura 9 mostra variação do desvio padrão para o mesmo intervalo.

Figura 9 - Variação do desvio padrão ao longo do tempo.

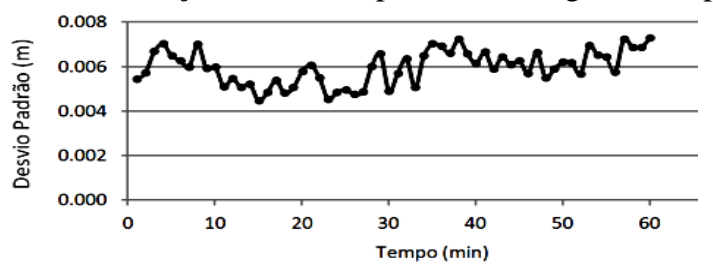

Bol. Ciênc. Geod., sec. Artigos, Curitiba, v. 21, n¹, p.126-148, jan-mar, 2015. 
O erro de zero foi calculado para todos os tempos de integração, desde $600 \mu \mathrm{s}$ até $4000 \mu \mathrm{s}$. Para todos os tempo de integração utilizados, o valor do Erro de Zero foi nulo (zero). Assim, a média amostral e o desvio padrão amostral também foram nulos (zero). Realizando um teste estatístico para testar a média populacional, com a curva $\mathrm{Z}$ (35 amostras), considerando como hipótese nula $\left(\mathrm{H}_{0}\right)$ de que a média do erro de zero é nulo $(m=0)$ para todos os tempo de integração possíveis, não foi possível rejeitar $\mathrm{H}_{0}$. Assim, desprezou-se o erro de zero.

O erro de escala, por sua vez, foi desprezado por dois motivos: (1) as medições são consideradas apenas dentro do intervalo de meio comprimento de onda $(7,5 \mathrm{~m})$, de modo que a alteração da frequência tem efeito em apenas um ciclo. (2) seria necessário que houvesse um erro da ordem de 2800 ppm na emissão da frequência para que gerasse um erro de $0,14 \mathrm{~mm}$ na medição e, frente a precisão da câmara, este valor pode ser desprezado.

A Figura 10 mostra um gráfico da diferença entre a distância medida em relação à distância de referência para os tempos de integração desde $600 \mu$ s até 4000 $\mu \mathrm{s}$. Os tempos de integração entre $100 \mu \mathrm{s}$ e $500 \mu$ s foram desconsiderados devido à iluminação precária (sub-iluminação). Observa-se que, quanto maior o tempo de integração, maiores são os erros associados. Três distâncias se destacam: 95 cm, 190 $\mathrm{cm}$ e $270 \mathrm{~cm}$. Nestas distâncias os erros foram os menores para todos os tempos de integração. Na posição $190 \mathrm{~cm}$, por exemplo, o erro médio para todos os tempos de integração é de $4,25 \mathrm{~cm}$, com desvio padrão de $0,36 \mathrm{~cm}$.

Figura 10 - Gráfico Erro vs. Distância teórica para todos os tempos de integração,

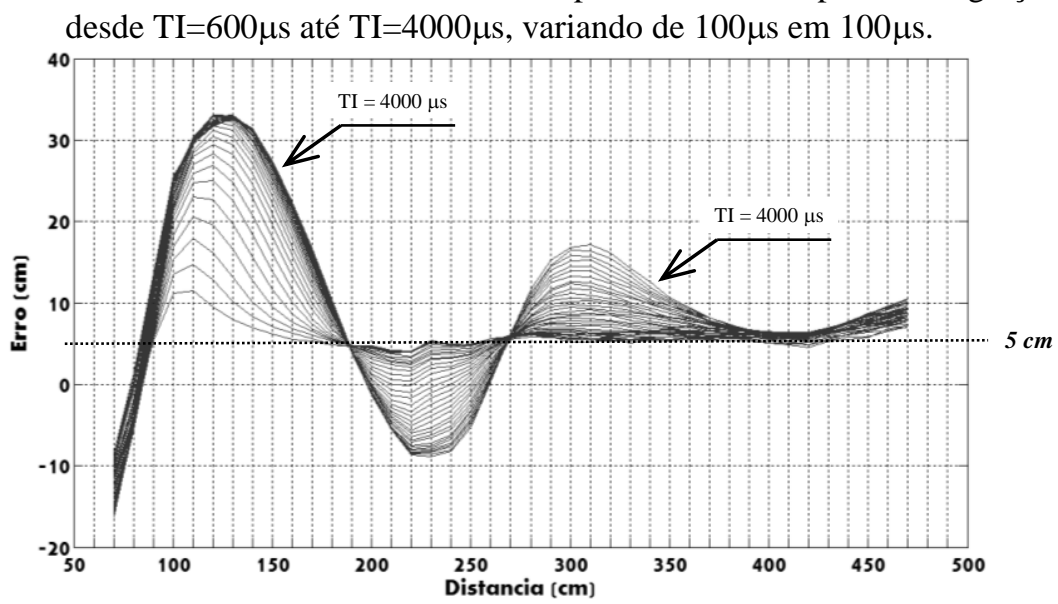

Na Figura 10 é evidente o comportamento cíclico da diferença das distâncias para todos os tempos de integração verificados, com a mesma frequência mas com amplitude variável em função do tempo de integração. 
A correção do erro cíclico fará com as amplitudes dos erros recaiam sobre uma linha horizontal distante $5 \mathrm{~cm}$ acima do eixo x. Este valor é a constante da equação de compensação do erro na distância, e é decorrente da impossibilidade de se determinar o centro de projeção do sensor.

Dividindo o gráfico da Figura $10 \mathrm{em}$ trechos, tem-se: (a) trechos onde as distâncias calculadas apresentam erros menores que $5 \mathrm{~cm}$ : entre $70 \mathrm{~cm}$ e $95 \mathrm{~cm}$ e entre $190 \mathrm{~cm}$ e $255 \mathrm{~cm}$; e (b) trechos onde as distâncias calculadas apresentam erros maiores que $5 \mathrm{~cm}$ : entre $95 \mathrm{~cm}$ e $190 \mathrm{~cm}$ e entre $255 \mathrm{~cm}$ e $470 \mathrm{~cm}$.

Também se observou que para tempos de integração entre $2000 \mu$ s e $4000 \mu$ s, entre as distâncias $70 \mathrm{~cm}$ e $190 \mathrm{~cm}$, as amplitudes dos erros apresentam valores similares. Desta forma entende-se que, neste intervalo de distâncias, é possível corrigir os erros cíclicos com uma mesma função, qualquer que seja o tempo de integração entre $2000 \mu$ s e $4000 \mu$ s. Para os outros casos, o erro deve ser modelado em função do tempo de integração.

Aplicando a Transformada Rápida Discreta de Fourier (DFFT) na série de erros medidos com tempo de integração $4000 \mu$ s, gerou-se o Espectro de Amplitudes da função no domínio da frequência, conforme é mostrado na Figura 11.

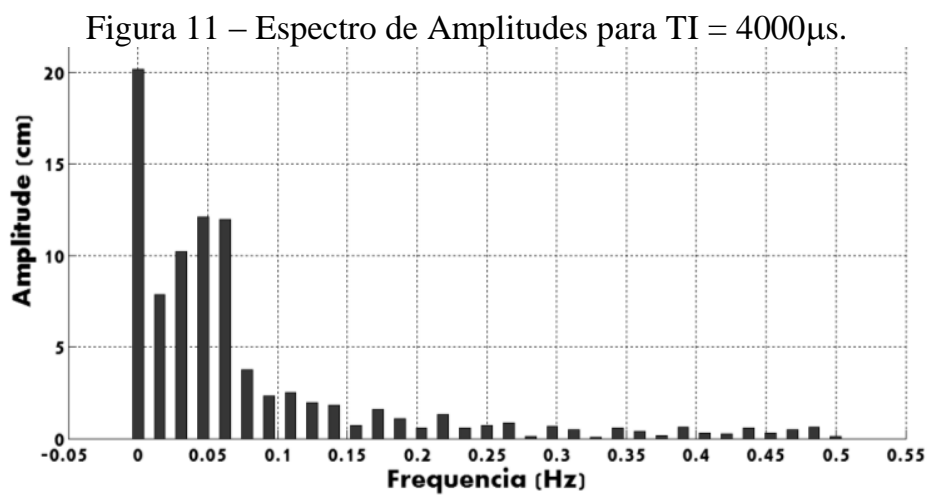

A frequência de maior amplitude tem valor $f=0,0469 \mathrm{~Hz}$. Logo, para o tempo de integração4000 $\mu$ s, é nesta frequência que a maior parcela do erro cíclico pode ser compensada. Para qualquer outro tempo de integração deve-se aplicar novamente a Transformada Rápida Discreta de Fourier para obter as suas respectivas frequências e escolher aquela de maior amplitude, que responde pela maior parte do erro cíclico. Pode-se adotar o valor de $5 \mathrm{~cm}$ para a constante $c$, conforme já fora mostrado na Figura 10. De maneira empírica escolheu-se o valor para a diferença de fase como sendo $\varphi=100$. Para modelar a amplitude variável, utilizou-se uma reta com coeficiente angular $\mathrm{m}=-1,2$, cruzando o eixo em $\mathrm{y}=38$ $(\mathrm{n}=38)$. Esta reta é mostrada na Figura 12. Assim, para TI $=4000 \mu \mathrm{s}$, tem-se as Equações 18 e 19 para compensar o erro cíclico devido a distância câmara-alvo. 
Silva, F. M. M ; Centeno, J. A. S.

$$
\mathrm{A}(\mathrm{x})=-1,2 \cdot \mathrm{x}+38
$$

$$
y(x)=5+A(x) \cdot \cos (2 \cdot \pi \cdot 0,0469 \cdot x+100)(19)
$$

Traçando um gráfico da equação dos erros para TI $=4000 \mu$ s e a equação modelada para TI $=4000 \mu$ s, tem-se a Figura 12 .

Figura 12 - Para TI = 4000 : Curva Erro vs. Distância, Curva modelada, e a Reta de coeficientes $\mathrm{m}=-1,2$ e $\mathrm{n}=38$ para modelar a Amplitude.

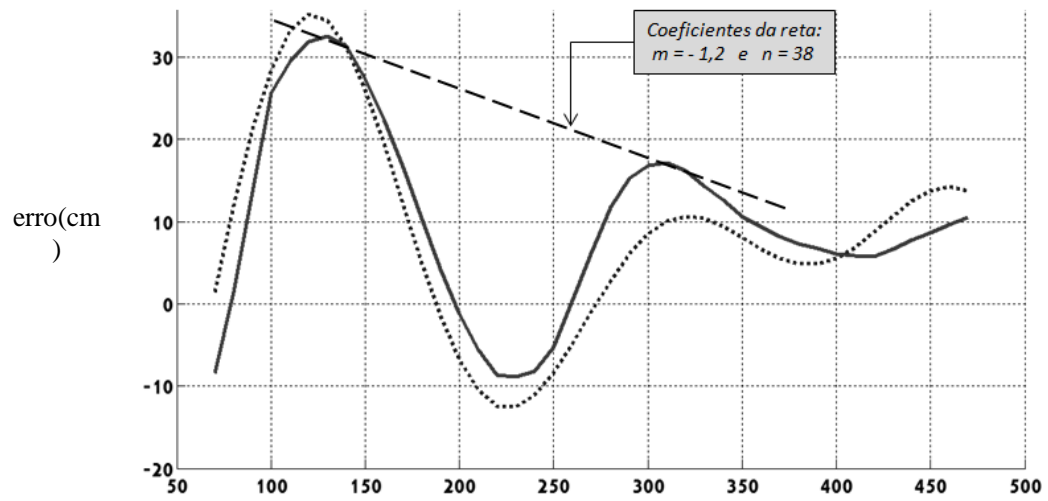

Curva Erro vs. Distância

-Eurva Modelada

-Retarpara amplitude variável

Observando as Figuras 13 e 14 percebe-se a melhora obtida nos valores de distância aplicando o modelo de correção proposto, que reduziu o erro cíclico.

Figura 13 - Relação da distância medida não corrigida e a distância teórica (de referência, medida pelo interferômetro) para TI $=4000 \mu \mathrm{s}$.

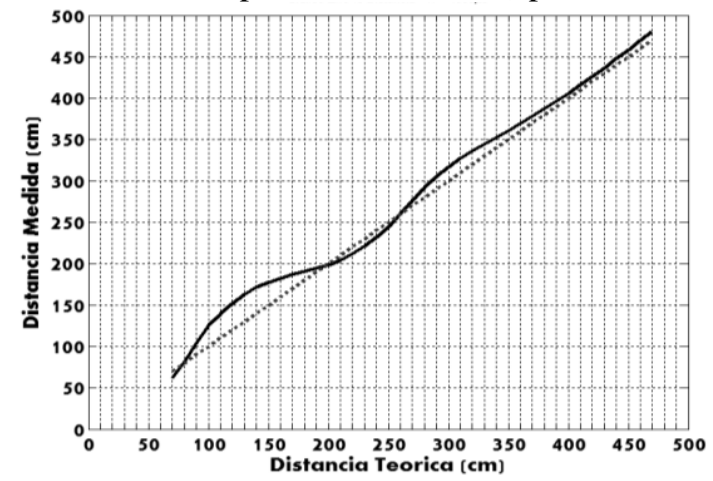

O erro médio quadrático (RMSE) antes da modelagem era de 15,01 cm (Figura 13), e após a modelagem diminuiu para $5,05 \mathrm{~cm}$ (Figura 14). 
Figura 14 - Relação da distância medida corrigida e a distância teórica (de referência, medida pelo interferômetro) para $\mathrm{TI}=4000 \mu \mathrm{s}$.

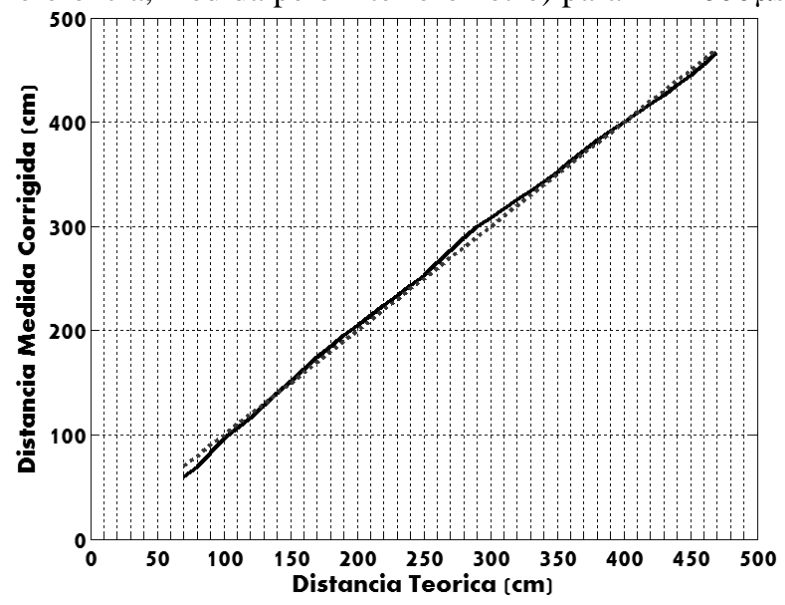

— Curva de distâncias medidas corrigidas

...Reta isenta de erros

\section{CONCLUSÕES}

O experimento realizado com diferentes distâncias e diferentes tempos de integração mostrou a existência de erros sistemáticos na medição de distâncias.

O erro é basicamente cíclico, sendo que os erros de zero e de escala puderam ser desprezados. Este erro cíclico apresentou a mesma frequência, mas com amplitudes variáveis para os tempos de integração a partir de $600 \mu \mathrm{s}$. Os tempos de integração abaixo de500 $\mu$ s foram desconsiderados devido à baixa iluminação captada pelo sensor. Conforme os tempos de integração foram aumentando, o comportamento cíclico dos erros ficou evidente, ao passo que o aumento das amplitudes dos erros garantiu à curva a forma mais senoidal / cossenoidal.

Para tempos de integração entre $2000 \mu$ s e $4000 \mu$ s, entre as distâncias 70 cm e $190 \mathrm{~cm}$, as amplitudes dos erros tiveram variação baixa. Assim, para distâncias pequenas e tempos de integração relativamente altos, apenas a distância teve influência significativa nas amplitudes dos erros.

A modelagem do erro utilizando a Transformada Rápida de Fourier obteve bons resultados, uma vez que para o tempo de integração $4000 \mu$ s houve diminuição do erro médio quadrático de $15,01 \mathrm{~cm}$ para $5,05 \mathrm{~cm}$.

Para trabalhos futuros sugere-se a utilização de outra função de modelagem da amplitude amortizada pelo aumento da distância.

\section{AGRADECIMENTOS}


Ao Conselho Nacional de Desenvolvimento Científico e Tecnológico (CNPq), pelo apoio financeiro que possibilitou esta pesquisa.

\section{REFERÊNCIAS BIBLIOGRÁFICAS}

ANDERSON, Dean; HERMAN, Herman; KELLY, Alonzo. Experimental characterization of commercial flash ladar devices. In Proceedings of International Conference on Sensing Technologies, New Zealand, November, 2005.

BECERRO, Frederic Garcia. External-Self-Calibration of a 3D time-of-fight camera in real environments. 51p. Dissertação (Mestre Erasmus Mundus em Visão e Robótica - Heriot-Watt University, Scotland, the Université de Borgogne, France, and the University of Girona, Spain, p. 06, 2008.

CHIABRANDO, Filiberto; CHIABRANDO, Roberto; PIATTI, Dario; RINAUDO, Fulvio. Sensors for 3D Imaging: Metric Evaluation and Calibration of a CCD/CMOS Time-of-Flight Camera. Sensors, v. 9, pp. 10080-10096, 2009.

FAGGION, Pedro Luiz. Obtenção dos elementos de calibração e certificação de medidores eletrônicos de distância em campo e laboratório. 134 p. Tese (Doutorado em Ciências Geodésicas) - Setor de Ciências da Terra,Universidade Federal do Paraná, Curitiba, 2001.

FALIE, Dragon; BUZULOIU, Vasile. Noise Characteristics of 3D Time-of-Flight Cameras. IEEE International Symposium on Signals, Circuits and Systems, p. 1 - 4, 13-14 July, Romenia, 2007.

FOIX, Sergi; ALENYA, Guillem; TORRAS, Carme. Lock-in time-of-flight (ToF) cameras: a survey.IEEE Sensors Journal, v. 11, n. 3, p. 1-11, 2011.

FUCHS, Stefan. Calibration and multipath mitigation for increased accuracy of time-of-flight camera measurements in robotic applications. 174 p. Tese (Doutorado em Engenharia). Faculdade de Engenharia Elétrica e Ciência da Computação, Universidade Tecnológica de Berlim, Berlim, 2012.

FUCHS, S.; HIRZINGER, G. "Extrinsic and depth calibration of ToFcameras," in Proceedings of the IEEE Conference on Computer Vision and Pattern Recognition, Alaska, v. 5, pp. 1-6, 2008.

HEBERT, Martial; KROTKOV, Eric. 3D measurements from imaging laser radars: How good are they? International Journal of Image and Vision Computing, Vol. 10, No. 3, pp. 170-178, April, 1992.

HUSSMANN, Stephan; HUHN, P.; HERMANSKI, A. Systematic distance deviation error compensation for a ToF-camera in the close-up range. In Proceedings of the IEEE International Instrumentation and Measurement Technology Conference, pp. 1546-1550, Austria, 2012.

HUSSMANN, Stephan; KNOLL, Florian. Modulation method for minimizing the depth distortion offset of lock-in TOF cameras. IEEE International Instrumentation and Measurement Technology Conference (I2mtc), 2013. 
HUSSMANN, Stephan; KNOLL, Florian; EDELER, Torsten. Modulation method including noise model for minimizing the wiggling error of TOF cameras. IEEE transactions on instrumentation and measurement, v. 63, n. 5, 2014.

KAREL, Wilfried. Integrated range camera calibration using image sequences from hand-held operations. The International Archives of Photogrammetry, Remote Sensing and Spatial Information Sciences, v. XXXVIII, Part 5, Beijing, 2008.

KHALMANN, Timo.; INGENSAND, Hilmar; REMONDINO, Fabio. Calibration for increased accuracy of the range imaging camera Swissranger ${ }^{\mathrm{TM}}$.ISPRS Archives - Volume XXXVI Part 5, ISPRS Commission V Symposium 'Image Engineering and Vision Metrology, Dresden, Germany, September p. 136141, 2006.

KHALMANN, Timo; REMONDINO, Fabio; GUILLAUME, Sébastien. Range imaging technology: new developments and applications for people identification and tracking. In Proceedings of Videometrics IX - SPIE-IS\&T Electronic Imaging, v. 6491, 2007.

KUHNERT, Klaus-Dieter; STOMMEL, Martin - Fusion of stereocamera and PMDcamera data for real-time suited precise 3D environment reconstruction. In Intelligent Robots and Systems (IROS), pp. 4780-4785, 2006.

KIM, Young Min; CHAN, Derek; THEOBALT, Christian; THRUN, Sebastian. Design and Calibration of a Multi-view TOF sensor fusion system. Computer Vision and Pattern Recognition Workshops, IEEE Computer Society Conference, p. 1-7, 2008.

KOLB, Andreas; BARTH, Erhardt; KOCH, Reinhard, LARSEN, Rasmus. Time-ofFlight Cameras in Computer Graphics. Computer Graphics Forum, V. 29, 141-149, 2010.

LANDGATE. Calibration of Eletronic Distance Measurement Instruments. Western Australian Land Information Authority. 2009. Disponível em: <http://www.landgate.wa.gov.au/docvault.nsf/web/PS_Survey_EDM_Calibrati on_Software/\$FILE/EDMCalibration.pdf >. Acesso em: fevereiro, 2013.

LANGE, Robert. 3D Time-of-Flight Distance Measurement with Custom SolidState Image Sensors in CMOS/CCD-Technology. 139p. Dissertation (Doctor of Technical Sciences). Department of electrical engineering and computer science at University of Siegen, Germany, 2000.

LICHTI, Derek. Self-calibration of a 3D range camera.The International Archives of the Photogrammetry, Remote Sensing and Spatial Information Sciences, V. XXXVII, Part B5, Beijing, 2008.

LICHTI, Derek, CHOW, Jacky C. K.; MITISHITA, E.; CENTENO, J.A.S.; SILVA, Felipe M. M.; BARRIOS, Roberto A.; CONTRERAS, Ilich. New Models for Scattering Bias Compensation in Time-of-Flight Range Camera Self Calibration.Jornal of Surveying Enginnering. American Society of Civil Engineers (ASCE), 2013. 
LINDNER, Marvin; KOLB, Andreas; RINGBECK, Thorsten. New Insights into the Calibration of ToF-Sensors. In Procedures $22^{\text {nd }}$ IEEE Conf. Comput. Vision Pattern Recognition, v. 1-3, pp. 1603-1607, 2008.

LINDNER, Marvin; KOLB, Andreas. Calibration of the intensity-related distance error of the PMD TOF-Camera. In: SPIE - Intelligent Robots and Computer Vision XXV, p. 6764-35, 2007.

LINDNER, Marvin; KOLB, Andreas - Lateral and Depth Calibration of PMDDistance Sensors. In ISVC, Ed. Springer, v. 2, p. 524-533, 2006.

LINDNER, Marvin. "Calibration and real-time processing of time-of-flight range data". Tese de Doutorado, Universidade de Siegen, Alemanha, 2010.

LINDNER, Marvin; SCHILLER, Ingo; KOLB, Andreas; KOCH, Reinhard. Time-of -flight sensor calibration for accurate range sensing.Journal Computer Vision and Image Understanding, v.114, issue 12, p. 1318-1328, Elsevier Science Inc. New York, USA, Dezembro, 2010.

LIU, Xinquiao. Cmos image sensors dynamic range and snr enhancement via statistical signal processing. 139p. Tese (Doutorado em Filosofia) Department of electrical engineering and the committee on graduate studies of Stanford University, USA, 2002.

MAIA, Tule César Barcelos. Estudo e análise de poligonais segundo a NBR 13.133 $e$ o sistema de posicionamento global. 175p. Dissertação (Mestrado em Transportes) - Escola de Engenharia de São Carlos, Universidade de São Paulo, São Carlos, 1999.

MAY, Stefan ; PERVOELZ, Kai; SURMANN, Hartmut. 3D Cameras: 3D Computer Vision of wide scope.Vision Systems: Applications, Edited by: Goro Obinata and Ashish Dutta, pp. 181-202, Vienna, Austria, 2007.

MAY, Stefan; FUCHS, Stefan; DROESCHEL, David; HOLZ, Dirk; NÜCHTER, Andreas. Robust 3D - Mapping with Time-of-Flight Cameras. International Conference on Intelligent Robots and Systems, p. 11-15, St. Louis, USA, 2009. MÖLLER, Tobias; KRAFT, Holger; FREY, Jochen; ALBRECHT, Martin; LANGE, Robert. Robust 3D measurement with PMD sensors. In: Proceedings of the 1st Range Imaging Research Day, ETH Zurich, Switzerland, 2005.

OLIVEIRA, Andrey Augusto Alves de. Modelagem tridimensional de superficies utilizando imagem ToF - estudo com a câmara PMD Camcube 2.0.127p.

Dissertação (Mestrado em Ciências Geodésicas) - Setor de Ciências da Terra, Universidade Federal do Paraná, Curitiba, 2011.

OLIVEIRA, Andrey Augusto Alves de; CENTENO, Jorge Antonio Silva; JUTZI, Boris. Tratamento de ruído em imagens de distância obtidas com a câmara PMD[VISION] ${ }^{\circledR}$ Camcube 2.0. III Simpósio Brasileiro de Ciências Geodésicas e Tecnologias da Geoinformação. Recife - PE, p. 001 - 007, 2730 de Julho de 2010

PIATTI, Dario. Time-of-Flight cameras: tests, calibration and multi-frame registration for automatic $3 D$ object reconstruction. 160 p. Tese. Doctoral school of Environment and Territory, Politecnico di Torino, Torino, 2010. 
PIATTI, Dario; RINAUDO, Fulvio. SR-4000 and CamCube3.0 Time of Flight (ToF) Cameras: Tests and Comparison.Remote Sensing. v. 4, p. 1069-1089, 2012.

RADMER, J; FUSTE, P.; SCHMIDT, H.; KRUGER, J. Incident light related distance error study and calibration of the PMD-range imaging camera. In Proceedings of the IEEE Conference on Computer Vision and Pattern Recognition. v. 1-3, pp. 1579-1584, Alaska, 2008.

REYNOLDS, Malcolm; DOBOS, Jozef; PEELY, Leto; WEYRICH, Tim, BROSTOW, Gabriel J. Capturing Time-of-Flight Data with Confidence. IEEE Conference on Computer Vision and Pattern Recognition (CVPR), 2011, p. 945 - 952, 20-25 June, 2011.

SABOV, Alexander; KRÜGER, Jörg. Identification and correction of flying pixels in range camera data. Spring Conference on Computer Graphics (SCCG), Slovakia, p. 135 - 142, 2008.

SHAHBAZI, Mozhdeh; HOMAYOUNI, Saeid; SAADATSERESHT, Mohammad; SATTARI, Mehran. Range camera self-calibration based on integrated bundle adjustment via joint setup with a 2D digital camera. Sensors, v. 11, p. 87218740, 2011.

STEIGER, OLIVIER; FELDER, JUDITH; WEISS, STEPHAN.CALIBRATION OF TIME-OF-FLIGHT RANGE IMAGING CAMERAS. Image Processing. 15th IEEE - ICIP - International Conference OnSAN DIEGO, CA, 2008.

SURVEYOR-GENERAL VICTORIA. EDM Calibration handbook - Edition 13. Published by the Victorian Government Department of Sustainability and Environment. Melbourne. 2012.

WIEDEMANN, Matthias; SAUER, Markus; DRIEWER, Frauke; SCHILLING, Klaus. Analysis and characterization of the PMD camera for application in mobile robotics. Proceedings of the 17th World Congress The International Federation of Automatic Control, Seoul, Korea, July 6-11, 2008.

(Recebido em março de 2014. Aceito em novembro de 2014). 